\title{
Deposição de filmes de TiN por gaiola catódica em atmosfera de plasma de $\mathrm{Ar}-\mathrm{N}_{2}-\mathrm{H}_{2}$
}

\author{
Deposition of TiN films in cathodic cage under plasma atmosphere of $\mathrm{Ar}-\mathrm{N}_{2}-\mathrm{H}_{2}$
}

\author{
Natália Daudt ${ }^{1}$; Barbosa, J. C. P. ${ }^{1}$; Macêdo, M. O. C ${ }^{1}$; Alves Jr.,C¹.
}

\begin{abstract}
Resumo
A gaiola catódica é uma técnica de nitretação/deposição desenvolvida no Labplasma (Laboratorório de Processamento de Materiais por Plama) na UFRN (Universidade Federal do Rio Grande do Norte) com intuito de reduzir os problemas indesejáveis da técnica convencional, principalmente a obtenção de um filme homogêneo. Conhecendo a aplicabilidade de filmes de TiN em janelas solares, o objetivo desse trabalho é obter um filme de TiN sobre um substrato de vidro utilizando a técnica de deposição por plasma através da gaiola catódica e estudar a influência das espécies ativas no plasma na deposição desse filme. Para tal, foi confeccionada uma gaiola catódica de titânio e utilizou-se uma atmosfera de $\mathrm{Ar}, \mathrm{N}_{2}$ e $\mathrm{H}_{2}$ nas seguintes fluxos em sccm: 4-3-0; 4-3-0,1 e 4-3-0,2; respectivamente, e o processo de deposição foi monitorado por Espectroscopia de Emissão Óptica (Optical Emission Spectroscopy-OES). Através das análises de difração de raios X, transmitância e microscopia óptica observou-se a formação de um filme de TiN em todas as condições, sendo que o filme foi mais espesso e homogêneo com aumento do fluxo de $\mathrm{H}_{2}$. Logo, uma pequena variação no fluxo de $\mathrm{H}_{2}$ modifica a quantidade das espécies ativas no plasma e consequentemente as características do filme depositado.
\end{abstract}

Palavras-chave: Gaiola catódica; Filme fino de TiN; Deposição a plasma.

\begin{abstract}
The cathodic cage plasma nitriding/deposition is an adaptation of ionic nitriding technique that was developed at Labplasma (Laboratório de Processamento de Materiais por Plasma) in UFRN (Universidade Federal do Rio Grande do Norte) in order to reduce undesirable problems of the conventional process, mainly obtaining a homogeneous film. TiN films are deposited on glass surface to be used as a clear solar control window. The purpose of this work is growing a TiN film on glass substrate using the cathodic cage plasma deposition technique and to study the influence of actives species of plasma in the growing of the film. In order to carry out the process, it has been constructed a cathodic cage of titanium. The titanium nitride was deposited on glass surface utilizing an atmosphere composed by argon, nitrogen and hydrogen in theses flows in sccm: 4-3-0; 4-3-0,1 and 4-3-0,2; respectively and the deposition process was monitored by Optical Emission Spectroscopy - OES. X-ray diffraction, transmittance and optical microscopy analysis show that a TiN film was formed in all conditions and that film was thicker and more homogeneous with increasing flow of $\mathrm{H}_{2}$. Therefore, a low variation in the flow of $\mathrm{H}_{2}$ modifies the quantity of actives species in plasma and consequently in the characteristics of the deposited film.
\end{abstract}

Keywords: Cahodic cage; TiN thin film; Plasma deposition. 


\section{Introdução}

Os filmes de nitreto de titânio são conhecidos por sua aplicação sobre aços como forma de aumentar a dureza superficial e de melhoria das propriedades anticorrosivas, sendo muito utilizados em ferramentas de corte ${ }^{(1)}$. Recentemente tem sido estudadas outras aplicações de filmes de TiN, explorando suas propriedades ópticas. Para tal, filmes de TiN são depositados sobre vidro a fim de serem utilizados em janelas com controle de luminosidade, visto que o TiN absorve seletivamente a luz solar, gerando economia de energia ${ }^{(2,3)}$.

A gaiola catódica é uma adaptação da nitretação iônica desenvolvida no Labplasma, com o intuito de diminuir os defeitos gerados pela técnica convencional. A gaiola catódica consiste em uma chapa cilíndrica com furos e uma tampa circular também com furos. Nessa configuração a gaiola funciona como catodo na qual é aplicada a diferença de potencial em relação às paredes da câmara. Desta maneira, os átomos arrancados através do processo de sputerring são da gaiola, de modo a evitar que a superficie da amostra sofra danos. Os átomos ejetados podem se combinar com o gás reativo da atmosfera do plasma e se depositar sobre a superfície da amostra ${ }^{(4,5)}$.

Essa técnica permite a obtenção de um filme mais homogêneo devido à diminuição do efeito de borda, que pôde ser comprovado por uma dureza uniforme em toda a amostra, além de diminuir a abertura de arcos e o efeito de cátodo oco na amostra ${ }^{(6,7)}$.

Utilizando a gaiola catódica, além de nitretar uma superfície metálica, é possível obter deposição de filmes, já que há deposição do material da gaiola, que pode ser combinado com as espécies ativas no plasma na superfície da amostra ${ }^{(8)}$.

Conhecendo a importância dos filmes de nitreto de titânio e sua grande aplicabilidade, foi confeccionada uma gaiola catódica de titânio para obtenção de filmes finos de nitreto de titânio em uma superfície de vidro. Para esse caso algumas dificuldades ocorrem devido à oxidação superficial da gaiola. Como o processo de deposição é causado pelo sputtering na superfície da gaiola, que ocorre preferencialmente nos seus furos, a presença desses óxidos dificulta o arrancamento dos átomos. Sendo assim, alguns cuidados devem ser tomados para evitar a contaminação da amostra e da gaiola pelos óxidos. Para tanto, foi feito uma etapa de limpeza da gaiola por plasma em uma atmosfera redutora.

Os filmes de TiN sobre uma superfície de vidro foram obtidos em diferentes composições de atmosfera do plasma. Com o intuito de investigar a influência das espécies ativas no plasma nas características do filme depositado, o processo de deposição foi monitorado através da Espectroscopia de Emissão Óptica (OES). O filme de TiN foi analisado por difração de raios $\mathrm{X}$ com ângulos de incidência rasante, microscopia óptica e transmitância.

\section{Materiais e Métodos}

Os materiais utilizados para deposição neste trabalho são amostras retangulares de vidro com $2 \mathrm{~mm}$ de espessura, $20 \mathrm{~mm}$ de comprimento e $20 \mathrm{~mm}$ de largura. As amostras foram limpas em um banho de acetona p.a. em ultrassom e foram secas utilizando uma corrente de ar quente $\left(60^{\circ} \mathrm{C}\right)$.

A câmara convencional de nitretação foi adaptada para configuração de deposição por gaiola catódica, que consiste em uma câmara de vácuo cilíndrica com $30 \mathrm{~cm}$ de diâmetro e $40 \mathrm{~cm}$ de altura, confeccionada em aço inoxidável, com uma janela de quartzo, a câmara é montada verticalmente e fechada utilizando flanges e junta de vedação tipo L; no flange inferior existem várias conexões: um sensor de pressão, entrada e saída de gás. A temperatura é medida por um termopar acoplado ao cátodo do reator. A fonte de tensão utilizada é contínua e possui voltagem e corrente máximas de $1.500 \mathrm{~V}$ e $2 \mathrm{~A}$, respectivamente. O fluxo de gases é controlado por meio de controladores de fluxo (MKS MFC1179A).

A gaiola utilizada foi confeccionada em titânio comercialmente puro, possui $70 \mathrm{~mm}$ de diâmetro, $42 \mathrm{~mm}$ de altura e $1 \mathrm{~mm}$ de espessura, com 45 furos de $8 \mathrm{~mm}$ de diâmetro, com distância entre os centros dos furos de 9,2 $\mathrm{mm}$. A tampa da gaiola possui diâmetro de $70 \mathrm{~mm}$ com 17 furos de $8 \mathrm{~mm}$, conforme a Fig. 1 .

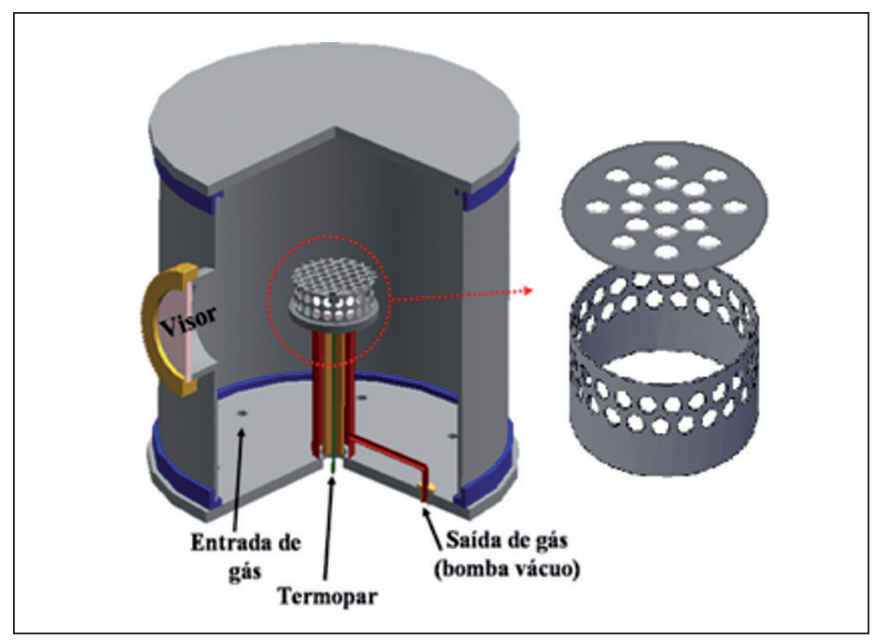

Figura 1. Representação esquemática em corte do reator de nitretação iônica com a configuração em gaiola catódica, mostrando detalhe da gaiola. 
A gaiola é posicionada sobre o cátodo. O plasma é formado na gaiola catódica, que funciona como cátodo (a parede da câmara é o ânodo), e não diretamente na superfície da amostra, que permanece em potencial flutuante, pois está posicionada em uma superfície isolante, que consiste em um disco de alumina.

As deposições foram realizadas em três diferentes atmosferas de plasma de $\mathrm{Ar}, \mathrm{N}_{2}$ e $\mathrm{H}_{2}$ nos seguintes fluxos (sccm), respectivamente: 4-3-0, 4-3-0,1 e 4-3-0,2; a $450^{\circ} \mathrm{C}$, por 120 minutos com a pressão constante de 2 mbar. Antes da deposição foi feita uma etapa de limpeza no reator de plasma utilizando uma atmosfera de $\mathrm{H}_{2}$ por 30 minutos na temperatura de $200^{\circ} \mathrm{C}$, na pressão de 1,2 mbar.

Os tratamentos de deposição foram monitorados por OES a fim de verificar as espécies ativas no plasma durante o processo de deposição. O sistema de espectroscopia óptica utilizado consiste em um espectrógrafo Ocean Optics USB 40000 com resolução óptica de 0,3 a $10 \mathrm{~nm}$, comprimento focal de $42 \mathrm{~mm}$ de entrada e $62 \mathrm{~mm}$ de saída e resposta óptica entre $200-1.100 \mathrm{~nm}$.

Os ensaios de difração de raios $\mathrm{X}$ foram realizados em um difratômetro Schimadzu XRD - 6000, com acessório para filmes finos. Foi utilizada a geometria Seeman-Bohlin, na qual o ângulo de incidência é rasante e fixo enquanto o detector faz a varredura 2q. Essa técnica é chamada de difração de raios X em ângulo rasante - GIXRD (Grazzing Incidence X-Ray Diffraction) e promove um significativo aumento no caminho ótico do feixe no interior do filme, de modo que a informação estrutural do difratograma é principalmente oriunda deste filme.

As medidas de transmitância foram feitas utilizando um espectrofotômetro Genesys 10 UV, com lâmpada de xenônio na faixa de comprimento de onda entre $190 \mathrm{e}$ $1100 \mathrm{~nm}$, com exatidão de $\pm 1 \mathrm{~nm}$, com leitura de transmitância de 0,3 a $125 \% \mathrm{~T}$.

As amostras foram observadas em um microscópio óptico Olympus, modelo BX60M com câmera Express - Series acoplada e a captura de imagem foi feita através do software Image Pro - Plus.

\section{Resultados e Discussão}

A Fig. 2 mostra o comportamento óptico de algumas espécies ativas do plasma em função do fluxo de $\mathrm{H}_{2}$.

Através do monitoramento do processo por OES notou-se que as espécies ativas no plasma influenciam diretamente nas características do filme crescido. Observou-se que com o aumento do fluxo de $\mathrm{H}_{2}$, houve um aumento na intensidade dos picos de $\mathrm{H} \alpha$ e $\mathrm{H} \beta$, uma diminuição na intensidade do pico de $\mathrm{N}_{2}$, e não houve um crescimento linear do pico de $\mathrm{N}^{2+}$. Os picos de $\mathrm{OH}$ são atribuídos ao arranchamento de partículas de oxigênio da gaiola que provavelmente reagem com o hidrogênio do plasma. $\mathrm{E}$ os picos de $\mathrm{CN}$ são atribuídos a contaminantes vindo do arranchamento de átomos da borracha de vedação do reator.

A Fig. 3 mostra os resultados de difração de raios $\mathrm{X}$ para as diferentes amostras com ângulo de incidência de $0,5^{\circ}$ e $2^{\circ}$.

Através da análise por GIXRD identificou-se dois picos referentes à fase d-TiN, indicando a deposição de um filme de nitreto de titânio sobre o substrato, que comprova a eficiência da técnica de deposição por gaiola catódica.

Notou-se que com o aumento do fluxo de hidrogênio tanto com o ângulo de incidência de $0,5^{\circ}$ como de $2^{\circ}$ houve um aumento na intensidade relativa dos picos de TiN. Isso está relacionado com a maior quantidade da fase d-TiN presente na amostra que é justificado por uma maior espessura do filme. Assim pode-se afirmar que com o aumento do fluxo de $\mathrm{H}_{2}$, houve um aumento na espessura do filme.

A Fig. 4 apresenta os resultados de transmitância.

Como se observou na análise de difração de raios X todos os filmes possuem a mesma fase, logo, pode-se afirmar que quanto menor a espessura do filme maior será sua transmitância. Dessa forma, os resultados de transmitância confirmam os resultados de difração de raios X. Assim, com o aumento do fluxo de $\mathrm{H}_{2}$, houve

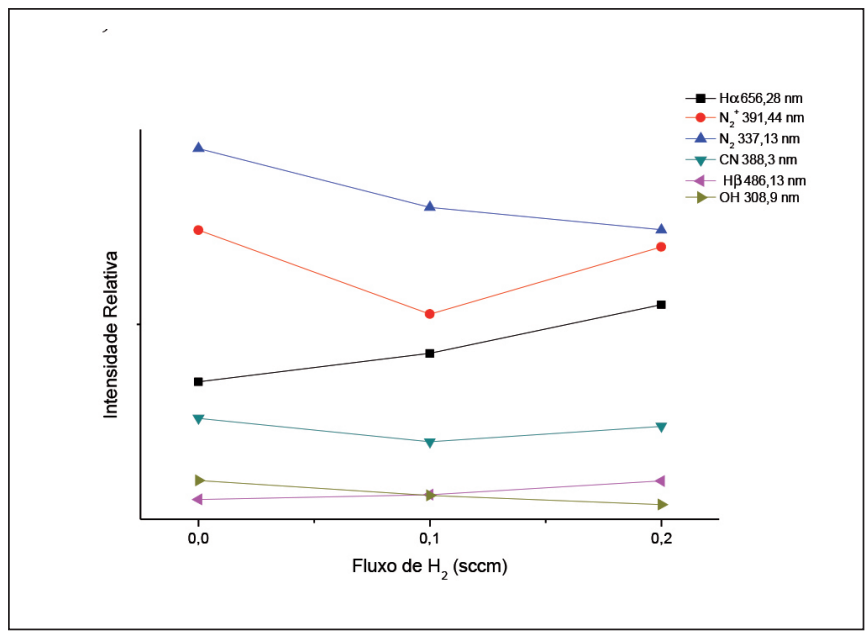

Figura 2. Valores da intensidade relativa de algumas espécies observadas no processo em função da variação do fluxo de $\mathrm{H}_{2}$. 


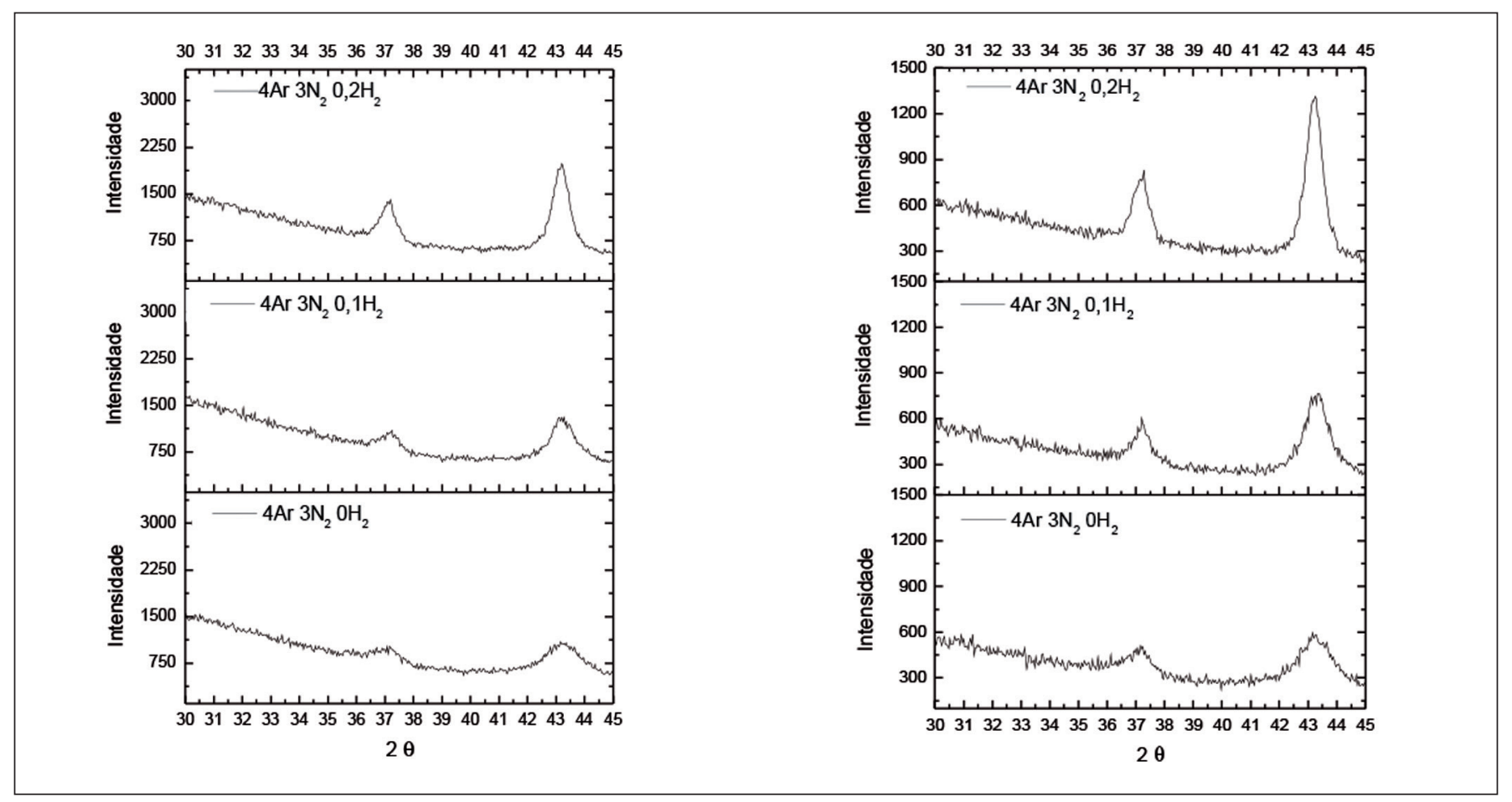

Figura 3. Difratograma com ângulo de incidência de (esquerda) $0,5^{\circ}$ e (direita) $2^{\circ}$ para o filme de TiN obtido nas diferentes atmosferas de plasma.

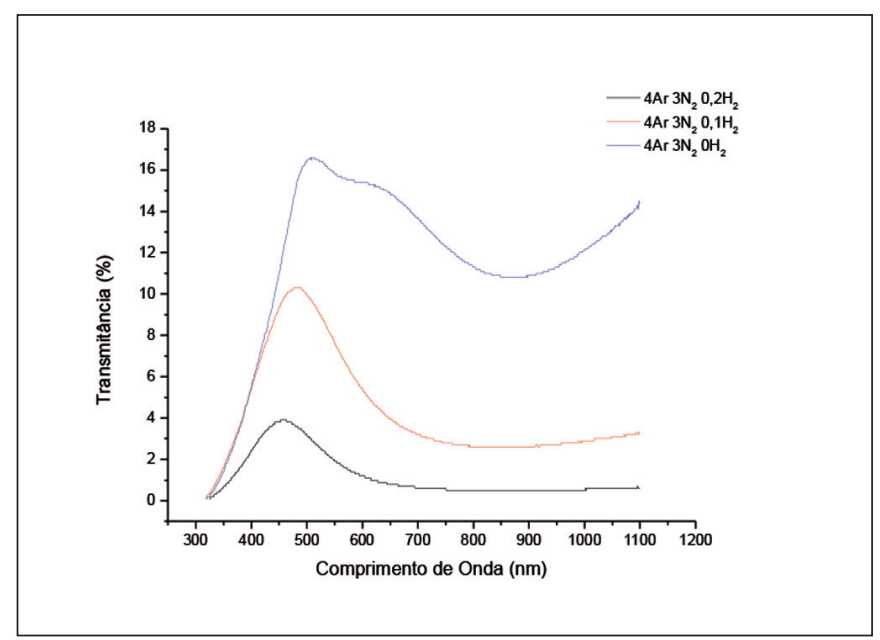

Figura 4. Transmitância das amostras obtidas nas diferentes composições da atmosfera do plasma.

diminuição da transmitância, indicando um aumento na espessura do filme.

A Fig 5 mostra as imagens obtidas por microscopia óptica.

Nas micrografias apresentadas na Fig. 4, a parte clara caracteriza a presença de filme e a parte escura caracteriza regiões onde não há filme, visto que a luz emitida pelo microscópio atravessou o vidro e encontrou o porta-amostra de cor preta. Constatou-se uma maior homogeneidade no filme obtido com maior fluxo de $\mathrm{H}_{2}$.

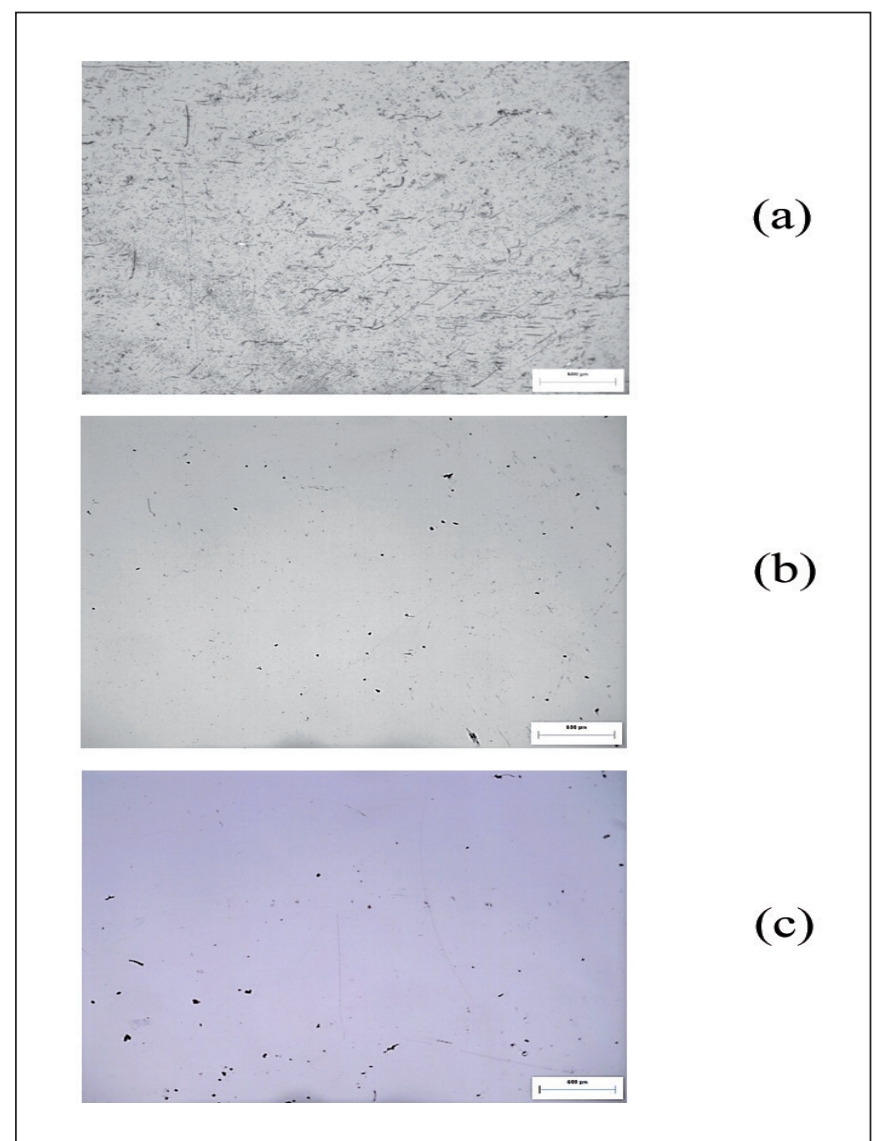

Figura 5. Microscopia óptica das amostras obtidas nos seguintes fluxos de $\mathrm{Ar}, \mathrm{N}_{2}$ e $\mathrm{H} 2$ respectivamente: (a) 4-3-0,2; (b) 4-3-0,1 e (c) 4-3-0 com aumento de 50x. 


\section{Conclusões}

É possível depositar um filme de nitreto de titânio em uma superfície de vidro utilizando um reator de plasma com a técnica de gaiola catódica.

Uma pequena variação no fluxo de $\mathrm{H}_{2}$ resulta em uma mudança significativa na intensidade relativa das espécies ativas no plasma, e numa variação na quantidade de filme

\section{Referências}

1. Veprk, S. "The search of novel, superhard materials".Journal of Vaccum Science and Technology A, Vol 17, p. 2401, 1999.

2. Uen, W. I.,et al. "Fabrication of low-resistivity and goldcolored TiN films by halide chemical vapor deposition with a low $\left[\mathrm{NH}_{3}\right] /\left[\mathrm{TiCl}_{4}\right]$ flow ratio". Thin Film Solids, Vol. 516, p. 99-103, 2007.

3. Tarniowy A., et al. "The effect of thermal treatment on the structure, optical and electrical properties of amorphous titanium nitride thin films". Thin Film Solids, Vol. 311, p. 93100, 1997.

4. Sousa, R. R. M. et al. "Nitriding in cathodic cage of stainless steel AISI 316: Influence of sample position". Vaccum, Vol. 83, p. 1402-1405, 2009. depositado, o que pode ser notado através da intensidade dos picos de difração de raios $\mathrm{X}$, bem como da \% de transmitância.

Quanto maior foi o fluxo de $\mathrm{H}_{2}$, maior a espessura do filme obtido. Ou seja, o filme mais espesso e mais homogêneo foi obtido em uma atmosfera de $\mathrm{Ar}, \mathrm{N}_{2}$ e $\mathrm{H}_{2}$ com os seguintes fluxos, respectivamente: 4-3-0,2 e o filme menos espesso na atmosfera de $\mathrm{Ar}, \mathrm{N}_{2}$ e $\mathrm{H}_{2}$ com os seguintes fluxos, respectivamente: 4-3-0.

5. Ahangarani, Sh., et al. "The influence of active screen plasma nitriding parameters on corrosion behavior of a low-alloy steel". Alloys and Compounds, Vol. 484, p. 222-229, 2009.

6. Gallo, S. C., Dong, H. "On the fundamental mechanisms of active screen plasma nitriding". Vaccum, Vol. 84, p. 321-325, 2010.

7. Hubbard, P. et al. "Investigation of nitrogen mass transfer within an industrial plasma nitriding system II: Application of a biased screen". Surface and coatings technology, Vol. 204, p. 1151-1157, 2010.

8, Araújo, F. O. et al. "Deposição de filme metálico em amostra de vidro em gaiola catódica". Revista Brasileira de Aplicações de Vácuo, Vol. 27, No. 3, p. 149-152, 2008. 